\title{
Fatores associados à prevalência de anomalias congênitas em nascidos vivos entre os anos de 2007-2017
}

\author{
Factors associated with the prevalence of congenital anomalies in live births between the \\ years 2007-2017 \\ Factores asociados a la prevalencia de anomalías congénitas en nacidos vivos entre los \\ años 2007-2017
}

Douglas Pereira Elizandro ${ }^{1 *}$, Márcia Dornelles Machado Mariot ${ }^{1}$, Fatima Helena Cecchetto ${ }^{1}$, Dayane de Aguiar Cicolella ${ }^{1}$, Camila da Rosa Maracci ${ }^{1}$.

\section{RESUMO}

Objetivo: Verificar os fatores associados a prevalência de anomalias congênitas em nascidos vivos entre os anos de 2007-2017 no município de Porto Alegre, Rio Grande do sul. Métodos: Este trabalho consiste em um estudo quantitativo, transversal e retrospectivo, realizado a partir de dados secundários disponíveis no Sistema de Informações sobre Nascidos Vivos. Foram incluídos no estudo nascidos vivos com alguma anomalia congênita descrita do município de Porto Alegre. A análise dos dados foi realizada através do programa SPSS. O estudo foi realizado de acordo com os aspectos éticos legais, respeitando as Diretrizes e Normas Reguladoras de Pesquisas Científicas do Conselho Nacional de Saúde através da Resolução № 466. Resultados: Os resultados demonstraram haver maior risco para ocorrência de anomalias congênitas para os nascidos vivos de mães com idade inferior a 19 anos e superior a 40 anos; com grau de instrução $<12$ anos; solteiras e separadas; de raça preta, parda ou indígena; para os prematuros, recém-nascidos do sexo masculino, de baixo peso e menores índices de Apgar. Conclusão: Considerando os achados desta pesquisa, espera-se incentivar o uso do banco de dados materno-infantis, pois conhecer os fatores associados poder ser útil no planejamento das ações dentro dos serviços de saúde.

Palavras-chave: Enfermagem, Anomalias congênitas, Epidemiologia.

\begin{abstract}
Objective: To verify the factors associated with the prevalence of congenital anomalies in live births between the years 2007-2017 in the city of Porto Alegre, Rio Grande do sul. Methods: This work consists of a quantitative, transversal and retrospective study, carried out from secondary data available in the Information System on Live Births. The study included live births with some congenital anomaly described in the city of Porto Alegre. Data analysis was performed using the SPSS program. The study was carried out according to legal ethical aspects, respecting the Guidelines and Regulatory Norms for Scientific Research of the National Health Council through Resolution No. 466. Results: The results showed that there is a greater risk for the occurrence of congenital anomalies for the live births of mothers under the age of 19 years and over 40 years; with education level $<12$ years; single and separated; black, brown or indigenous; for premature infants, male newborns, underweight and lower Apgar scores. Conclusion: Considering the findings of this research, it is expected to encourage the use of the maternal and child database, as knowing the associated factors can be useful in planning actions within health services.
\end{abstract}

Keywords: Nursing, Congenital anomalies, Epidemiology.

${ }^{1}$ Centro Universitário Cesuca (CESUCA), Cachoeirinha - RS.

*E-mail: contatodouglas.elizandro@outlook.com 


\section{RESUMEN}

Objetivo: Verificar los factores asociados a la prevalencia de anomalías congénitas en nacidos vivos entre los años 2007-2017 en la ciudad de Porto Alegre, Rio Grande do sul. Métodos: Este trabajo consiste en un estudio cuantitativo, transversal y retrospectivo, realizado a partir de datos secundarios disponibles en el Sistema de Información de Nacidos Vivos. El estudio incluyó nacidos vivos con alguna anomalía congénita descrita en la ciudad de Porto Alegre. El análisis de los datos se realizó mediante el programa SPSS. EI estudio se llevó a cabo de acuerdo con los aspectos éticos legales, respetando los Lineamientos y Normas Reglamentarias para la Investigación Científica del Consejo Nacional de Salud mediante Resolución No. 466. Resultados: Los resultados mostraron que existe un mayor riesgo de ocurrencia de anomalías congénitas para los nacidos vivos de madres menores de 19 años y mayores de 40 años; con un nivel educativo $<12$ años; soltero y separado; negro, marrón o indígena; para recién nacidos prematuros, recién nacidos varones, bajo peso y puntuaciones de Apgar más bajas. Conclusión: Teniendo en cuenta los hallazgos de esta investigación, se espera incentivar el uso de la base de datos materno-infantil, ya que conocer los factores asociados puede ser útil para planificar acciones dentro de los servicios de salud.

Palabras clave: Enfermería, Anomalías congénitas, Epidemiología.

\section{INTRODUÇÃO}

As Anomalias Congênitas (AC) é a segunda maior causa de mortalidade infantil a nível mundial. No continente Americano, cerca de uma a cada dez mortes ocorrem devido a estas anomalias (OMS, 2019). Segundo a Organização Mundial da Saúde (OMS) (2019) as AC podem ser conceituadas como quaisquer alterações estruturais ou funcionais da evolução fetal, no qual seu princípio é multifatorial e complexo, podendo ser de fatores genéticos, ambientais, socioeconômicos ou origem desconhecida. Levando em consideração que sua causa muitas vezes não é diagnosticada, acaba trazendo mais dificuldades para criação de métodos profiláticos para evitá-las (OMS, 2019).

Uma grande parte da mortalidade infantil está associada as anomalias congênitas, a principal maneira de identificar os casos é através de informações dos sistemas de saúde. Portanto, as anomalias congênitas (AC) são responsáveis por cerca de um a cada dez óbitos de crianças com a idade inferior a 5 anos, sendo a segunda principal causa de mortalidade a nível mundial, ficando atrás apenas da prematuridade (MINISTÉRIO DA SAÚDE, 2019; OMS, 2019;)

A mortalidade em crianças menores de 5 anos que também pode ser chamada de mortalidade infantil, é um dos indicadores acompanhados pela Organização das Nações Unidas (ONU) e sua redução está dentro dos Objetivos de Desenvolvimento do Milênio (ODM) para o período de 1990 a 2015 (MINISTÉRIO DA SAÚDE, 2019). A taxa mortalidade infantil (TMI) caracteriza um importante indicador de saúde, pois está ligado diretamente na qualidade da assistência prestada e consequentemente permitindo com que se veja o grau de desenvolvimento nas questões de saúde pública de uma determinada região. A TMI é calculada através do número de mortes de crianças menores de um ano, sobre o número de nascidos vivos (multiplicada por 1000) e demonstra o risco de um nascido vivo vir a evoluir para um óbito (FRANÇA E e LANSKY S, 2016; LIMA L, et al., 2017).

As anomalias podem acontecer em todos os órgãos e aparelhos do corpo, porém as principais no Brasil são as malformações comuns dos membros (polidactilia, sindactilia, etc.), seguidos do sistema nervoso (espinha bífida, hidrocefalia, etc.) e do coração (MINISTÉRIO DA SAÚDE, 2018). Cabendo ressaltar que existem também algumas anomalias que são incompatíveis com a vida, como, por exemplo, a anencefalia que se caracteriza pela ausência total ou parcial do encéfalo. Aproximadamente $80 \%$ dos casos são natimortos, ou seja, morreram ainda no útero, os demais morrem após horas ou dias (MINISTÉRIO DA SAÚDE, 2014).

As cardiopatias são as anomalias com maior incidência entre os nascidos vivos, dados epidemiológicos apresentam uma prevalência de 6-12 por 1.000 nascidos vivos em todo mundo (AMERICAN HEART 
ASSOCIATION, 2014). Os dados epidemiológicos do Brasil, segundo o Sistema de Informações sobre Nascidos Vivos (SINASC) demonstram que aconteceram aproximadamente 20.227 mil nascimentos de neonatos com anomalias congênitas do aparelho cardiovascular (MINISTÉRIO DA SAÚDE, 2017).

O estado do Rio grande do Sul registrou cerca de 1.700 anomalias ambos no período linear de 10 anos iniciando em 2007 e finalizando 2017, o município de Porto Alegre, foi responsável por 1.241 casos. A compreensão do cenário de anomalias congênitas é de extrema relevância na saúde pública, pois, permitirá, mediante a planejamento a elaboração de estratégias de melhorias assistenciais e que auxiliem na redução da mortalidade por causas evitáveis (MINISTÉRIO DA SAÚDE, 2017).

A compreensão do cenário de anomalias congênitas é de extrema relevância na saúde pública, pois, permitirá, mediante a planejamento a elaboração de estratégias de melhorias assistenciais e que auxiliem na redução da mortalidade por causas evitáveis. Levando em consideração estes dados, o estudo se torna relevante, pois analisar este indicador pode trazer resultados que auxiliem na criação de planejamento e intervenções dentro de um município. O presente estudo terá como objetivo os fatores associados a prevalência de anomalias congênitas em nascidos vivos entre os anos de 2007-2017 no município de Porto Alegre, Rio Grande do sul.

\section{MÉTODOS}

Este trabalho consiste em um estudo quantitativo, transversal e retrospectivo, em que foi realizada uma análise de dados secundários a partir do SINASC. A pesquisa quantitativa é definida por Fonseca JJ (2002) como dados que podem ser quantificados, diferentemente da pesquisa qualitativa este tipo de estudo se centra na objetividade.

Para realização do presente estudo foram avaliados os dados disponíveis no SINASC. Os critérios de inclusão utilizados constituíram-se de; nascidos vivos que possuam anomalias congênitas descritas; nascidos entre os anos de 2007 - 2017; pertencentes ao município de Porto Alegre. Serão excluídos os registros que estiverem incompletos no banco de dados.

Para análise estatística da pesquisa, os dados foram agrupados em um banco de dados no software Microsoft Excel 2013, posteriormente foram exportados ao programa de informática SPSS Statistics para Windows, versão 21.0. Nos casos de associação entre variáveis, utilizou-se a análise bivariada, através do teste quiquadrado, além da razão de prevalência para medir a força da associação entre as variáveis estudadas, com intervalo de confiança de $95 \%$ e o nível de significância de 5,0\%. O modelo de regressão linear foi utilizado para estimar a incidência de anomalias congênitas ao longo do tempo. Para os procedimentos descritivos, foram apresentadas as frequências absolutas e relativas (frequências e percentuais) e também a apresentação gráfica das informações, através de tabelas e quadros.

O presente estudo seguiu os aspectos éticos legais, respeitando as Diretrizes e Normas Reguladoras de Pesquisas Científicas do Conselho Nacional de Saúde através da Resolução № 466 de 12 de dezembro de 2012. O projeto de pesquisa foi enviado para apreciação do Comitê de Ética de pesquisa da Faculdade Cesuca com o intuito de obter aval ético e metodológico para o desenvolvimento do trabalho, tendo sido aprovado sob CAAE: 36873120.5 .0000 .5665$.

\section{RESULTADOS}

$\mathrm{Na}$ cidade de Porto Alegre, Rio grande do Sul, foram registrados 207.527 nascidos vivos consultadas através das do sistema de informação em saúde SINASC. Conforme os dados apresentados na Tabela 1, referentes ao perfil sociodemográfico materno, foi observado que há uma maior frequência de anomalia congênita em gestantes que se encontram na faixa etária dos 10 a 19 anos $(1,75 \%)$, seguida da faixa etária 40 ou mais (2,19\%). Quanto ao grau de instrução, as mulheres com menos de 12 anos de estudo foram as que mais incidiram os casos, representando, respectivamente 3,53\%, 1,90\% e 1,63\%.

A situação marital foi relevante em mulheres solteiras (1,67\%) e separadas $(2,17 \%)$, enquanto o critério de raça apresentou como fatores de risco indígenas $(3,32 \%)$ pardos $(1,73 \%)$ e pretos $(1,83 \%)$. Cabendo 
destacar, ainda, quanto aos fatores sócios demográficos estudados, que os extremos de faixa etária (10-19 e de 40 ou +), grau de instrução (inferior a 12 anos), situação conjugal (solteira ou separada) e as raças (preta, parda ou indígena) demonstraram estar associadas, de forma estatisticamente significativa $(p<0,001)$, com a ocorrência da anomalia congênita.

Tabela 1 - Perfil sociodemográfico materno conforme o SINASC.

\begin{tabular}{|c|c|c|c|}
\hline \multirow{2}{*}{ Variáveis } & \multicolumn{2}{|c|}{ Anomalia Congênita } & \multirow[t]{2}{*}{$\mathbf{P}$} \\
\hline & $\mathbf{N}$ & $\%$ & \\
\hline Faixa etária & & & $<0,001$ \\
\hline 10 a 19 anos & 523 & $1,75^{*}$ & \\
\hline 20 a 24 anos & 716 & 1,58 & \\
\hline 25 a 29 anos & 666 & 1,43 & \\
\hline 30 a 34 anos & 690 & 1,45 & \\
\hline 35 a 39 anos & 448 & 1,48 & \\
\hline 40 anos ou mais & 177 & $2,19^{*}$ & \\
\hline \multicolumn{4}{|l|}{ Grau de instrução } \\
\hline Nenhuma & 18 & $3,53^{*}$ & \\
\hline De 1 a 8 anos & 856 & $1,90^{*}$ & \\
\hline De 9 a 11 anos & 1597 & $1,63^{*}$ & \\
\hline 12 anos ou mais & 735 & 1,16 & \\
\hline Ignorado & 14 & $4,58^{*}$ & \\
\hline Situação marital & & & $<0,001$ \\
\hline Solteira & 2084 & $1,67^{*}$ & \\
\hline Casada/União estável & 1049 & 1,33 & \\
\hline Viúva & 5 & 1,89 & \\
\hline Separada & 69 & $2,17^{*}$ & \\
\hline Ignorado & 13 & $4,38^{*}$ & \\
\hline Cor/Raça & & & $<0,001$ \\
\hline Branca & 2316 & 1,47 & \\
\hline Preta & 515 & $1,83^{*}$ & \\
\hline Amarela & 2 & 0,93 & \\
\hline Parda & 370 & $1,73^{*}$ & \\
\hline Indígena & 8 & $3,32^{*}$ & \\
\hline Ignorada & 9 & 2,68 & \\
\hline
\end{tabular}

Nota: *Associação estatisticamente significativa pelo teste dos resíduos ajustados a 5\% de significância. Fonte: Elizandro DP, et al., 2021; dados extraídos do SINASC.

Quanto aos dados neonatais, os fatores que obtiveram relevância nos dados coletados foram a duração da gestação, números de consultadas de pré-natal realizadas, sexo, peso e Apgar. A análise mostrou que a prematuros $(3,05 \%)$ apresentam maior risco associado a anomalias congênitas $(p<0,001)$. $O$ tipo de parto não demostrou correlacionar-se a ocorrência de malformações, entretanto, ressalta-se que o $p$ valor foi limítrofe 
$(p=0,051)$. Observando a variável de realização de pré-natal, a não realização do $P N(p=0,013)$, e o um número de consultas igual ou menos a seis $(p<0,001)$ revelou que gestantes com neonatos acometidos por anomalias congênitas tem menor adesão ao acompanhamento de pré-natal.

A variável sobre o tipo de gestação, se de feto único ou de múltiplos, não demonstrou associar-se a ocorrência de anomalias $(p=0,384)$. Nascidos vivos com baixo peso a nascer $(p<0,001)$ e com o escore de Apgar abaixo de $8(p<0,001)$ foram os mais acometidos por defeitos congênitos. No quesito sexo, o masculino se mostrou como um fator de risco em relação ao feminino tendo maior frequência $(p<0,001)(n=1836 / 3220)$, conforme demonstra a Tabela 2.

Tabela 2 - Dados de pré-natal nascidos vivos com anomalias congênitas conforme SINASC.

\begin{tabular}{|c|c|c|c|}
\hline \multirow{2}{*}{ Variáveis } & \multicolumn{2}{|c|}{ Anomalia Congênita } & \multirow[t]{2}{*}{$\mathbf{P}$} \\
\hline & $\mathbf{N}$ & $\%$ & \\
\hline Duração da gestação & & & $<0,001$ \\
\hline$<37$ semanas & 719 & $3,05^{*}$ & \\
\hline 37 a 41 semanas & 2480 & 1,36 & \\
\hline 42 ou mais & 19 & 1,3 & \\
\hline Ignorado & 2 & 1,56 & \\
\hline Tipo de parto & & & 0,051 \\
\hline Vaginal & 1520 & 1,49 & \\
\hline Cesáreo & 1700 & 1,62 & \\
\hline Realização de pré-natal & & & 0,013 \\
\hline Não & 98 & $2,02^{*}$ & \\
\hline Sim & 3115 & 1,54 & \\
\hline Ignorado & 7 & 2,4 & \\
\hline Número de consultas & & & $<0,001$ \\
\hline 1 a 3 consultas & 254 & $1,87^{*}$ & \\
\hline 4 a 6 consultas & 695 & $1,79^{*}$ & \\
\hline 7 ou mais consultas & 2166 & 1,44 & \\
\hline Ignorado & 7 & 2,4 & \\
\hline Não realizou & 98 & $2,02^{*}$ & \\
\hline Tipo de Gestação & & & 0,384 \\
\hline Única & 3133 & 1,55 & \\
\hline Dupla & 87 & 1,61 & \\
\hline Tripla ou mais & 0 & 0 & \\
\hline Peso do bebê & & & $<0,001$ \\
\hline$<500 \mathrm{~g}$ a $2.499 \mathrm{~g}$ & 713 & $3,50^{*}$ & \\
\hline $2.500 \mathrm{~g}$ a $3.999 \mathrm{~g}$ & 2369 & 1,35 & \\
\hline $4.000 \mathrm{~g}$ ou mais & 138 & 1,27 & \\
\hline Apgar 5 minuto & & & $<0,001$ \\
\hline 0 a 5 & 193 & $13,5^{*}$ & \\
\hline 6 a 7 & 231 & $5,90^{*}$ & \\
\hline 8 a 10 & 2776 & 1,38 & \\
\hline Ignorado & 20 & 2,23 & \\
\hline
\end{tabular}

Nota: *Associação estatisticamente significativa pelo teste dos resíduos ajustados a $5 \%$ de significância.

Fonte: Elizandro DP, et al., 2021; dados extraídos do SINASC. 
Durante os anos de 2007-2017 foram notificadas 3.220 anomalias em 207.527 nascidos vivos. A incidência de casos durante este período é de 1,55\% (Figura 1). O modelo de Regressão Linear foi estatisticamente significativo, indicando uma tendência a queda na incidência de anomalia congênita ao longo do tempo. A cada ano transcorrido o percentual de anomalia congênita reduz $0,045 \%(p=0,014)$.

Figura 1 - Incidência de anomalias congênitas.

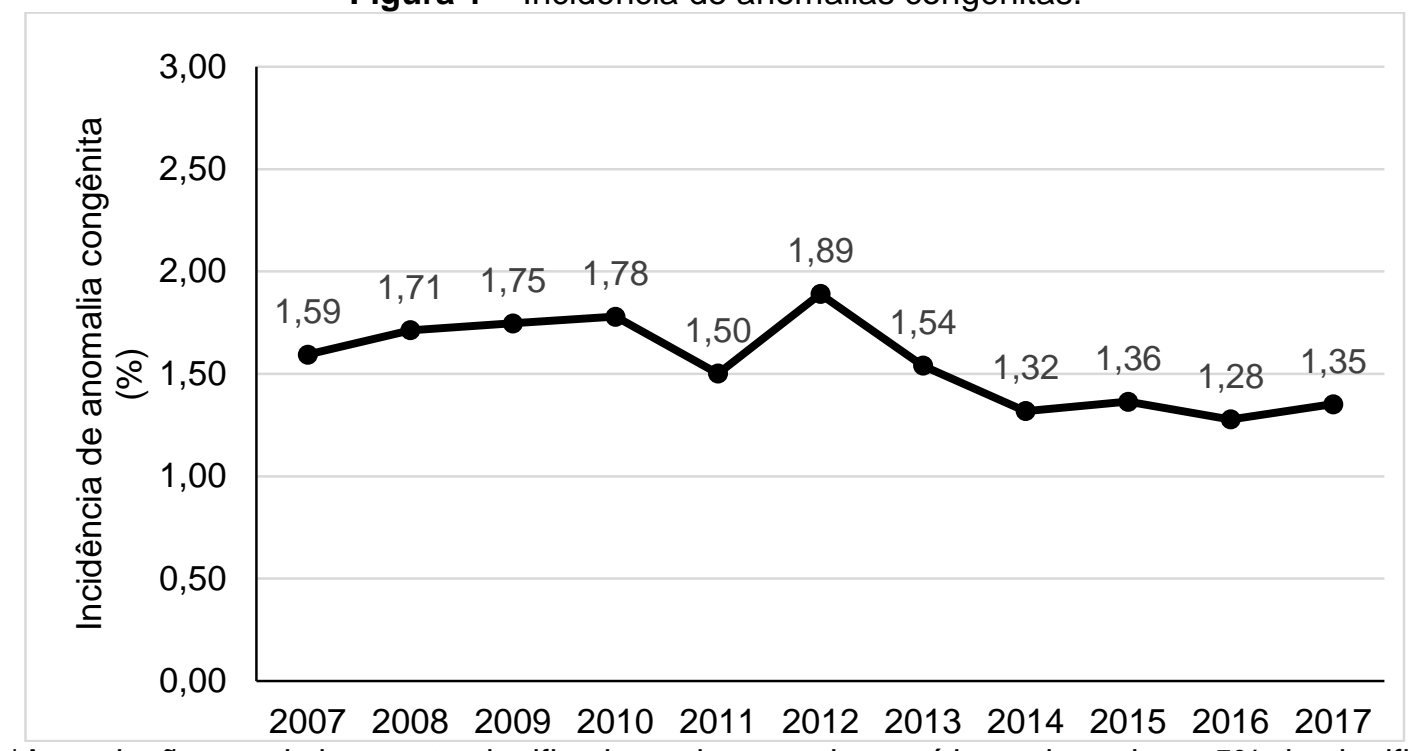

Nota: *Associação estatisticamente significativa pelo teste dos resíduos ajustados a $5 \%$ de significância.

Fonte: Elizandro DP, et al., 2021; dados extraídos do SINASC.

Referente aos tipos de anomalias, as mais frequentes ao longo dos anos estudados foram: as malformações do aparelho osteomuscular (20,52\%), seguidas das anomalias de malformações do sistema circulatório $(16,48 \%)$, malformações no sistema geniturinário $(9,00 \%)$ e deformidades congênitas dos pés $(7,23 \%)$. Outras malformações congênitas não especificadas no SINASC representam $(19,31)$ e a soma das demais $27,86 \%$, conforme demonstra a (Tabela 3 ).

Tabela 3 - Prevalência das anomalias congênitas no município de Porto Alegre entre 2007-2017 de acordo com dados do SINASC.

\begin{tabular}{lcc}
\hline Condição & Número de Casos & Prevalência \\
\hline Outras malformações aparelho osteomuscular & 661 & $20,52 \%$ \\
Outras malformações congênitas & 622 & $19,31 \%$ \\
Malformações sistema circulatório & 518 & $16,08 \%$ \\
Outras malformações aparelho geniturinário & 290 & $9,00 \%$ \\
Deformidade congênitas nos pés & 233 & $7,23 \%$ \\
Anomalias cromossômicas NCOP & 167 & $5,18 \%$ \\
Fenda labial e fenda palatina & 159 & $4,93 \%$ \\
Malformações sist. nervoso & 155 & $4,81 \%$ \\
Espinha bífida & 99 & $3,07 \%$ \\
Outras malformações aparelho digestivo & 83 & $2,57 \%$ \\
Hemangioma e linfangioma & 81 & $2,51 \%$ \\
Testículo não descido & 74 & $2,29 \%$ \\
Deformidade congênitas no quadril & 37 & $1,14 \%$ \\
Sem anomalia congênita/não informado & 31 & $0,96 \%$ \\
Ausência atresia e estenose do intestino delgado & 10 & $0,40 \%$ \\
\hline
\end{tabular}

Legenda: NCOP: Não classificáveis em outras partes.

Nota: *Associação estatisticamente significativa pelo teste dos resíduos ajustados a $5 \%$ de significância.

Fonte: Elizandro DP, et al., 2021; dados extraídos do SINASC. 
Na Tabela 4 apresentamos os principais tipos de anomalias congênitas estratificadas por sexo, dentre elas, as anomalias como fenda labial e fenda palatina, testículo não descido, malformações do aparelho geniturinário, foram mais prevalentes no sexo masculino. Enquanto no sexo feminino podemos observar maior significância nas malformações do aparelho circulatório, deformidades congênitas no quadril, deformidades congênitas no aparelho osteomuscular e em outras malformações congênitas não especificadas no SINASC.

Após análise de dados sobre os tipos de anomalias, percebeu-se que em algumas delas, o sexo do bebê e fator protetor ou de risco, anomalias como mal formações congênitas no quadril e mal formações do sistema circulatório, foram mais frequentes no sexo feminino, enquanto fenda labial, fenda palatina e mal formações no aparelho geniturinário acometeram mais o sexo masculino.

Tabela 4 - Anomalias congênitas por sexo.

\begin{tabular}{lcccc}
\hline \multirow{2}{*}{ Anomalia } & \multicolumn{2}{c}{ Masculino } & \multicolumn{2}{c}{ Feminino } \\
\cline { 2 - 5 } & $\mathbf{N}$ & $\%$ & $\mathbf{N}$ & $\%$ \\
\hline Espinha bífida & 51 & 2,8 & 48 & 3,5 \\
Outras malformações congênitas do sistema nervoso & 89 & 4,8 & 65 & 4,8 \\
Malformações congênitas do aparelho circulatório & 253 & 13,8 & 262 & $19,2^{*}$ \\
Fenda labial e fenda palatina & 105 & $5,7^{*}$ & 53 & 3,9 \\
Ausência atresia e estenose do intestino delgado & 5 & 0,3 & 5 & 0,4 \\
Outras malformações congênitas aparelho digestivo & 41 & 2,2 & 40 & 2,9 \\
Testiculo não-descido & 74 & $4,0^{*}$ & 0 & 0,0 \\
Outras malformações do aparelho geniturinário & 243 & $13,2^{*}$ & 34 & 2,5 \\
Deformidades congênitas do quadril & 11 & 0,6 & 26 & $1,9^{*}$ \\
Deformidades congênitas dos pés & 128 & 7,0 & 105 & 7,7 \\
Outras malformação e deformação congênita & 355 & 19,3 & 306 & $22,5^{*}$ \\
aparelho osteomuscular & 334 & 18,2 & 287 & $21,1^{*}$ \\
Outras malformações congênitas & 38 & 2,1 & 43 & 3,2 \\
Hemangioma e linfangioma & 19 & 1,0 & 12 & 0,9 \\
Sem anomalia congênita/não informado & & & & 0.3 \\
\hline
\end{tabular}

Nota: *Associação estatisticamente significativa pelo teste dos resíduos ajustados a 5\% de significância.

Fonte: Elizandro DP, et al., 2021; dados extraídos do SINASC.

\section{DISCUSSÃO}

Os resultados encontrados nesta pesquisa apontam uma associação entre a idade da mãe e a ocorrência de anomalias congênitas. A literatura destaca a idade materna como um fator de risco para a ocorrência e desenvolvimento de anomalias congênitas. No caso de mães com idade precoce, há um risco elevado de haver malformações do feto provocados por fatores externos como o uso indevido de medicações para induzir o aborto e o uso do álcool (MENDES CM, et al., 2018).

Demonstra-se também, um grau elevado para gestantes com uma maior idade materna, visto que algumas pesquisas pontuam que as doenças crônicas pré-existentes, como por exemplo hipertensão arterial sistêmica e diabetes mellitus estão relacionadas as más formações congênitas, apesar de ainda estar indefinido se a anomalia está relacionada a doença crônica ou ao tratamento contínuo (BATEMAN BT, et al., 2015). Estes dados vão de encontro aos achados desta pesquisa, que demostrou que os extremos da idade materna, estão relacionados a prevalência de anomalias congênitas.

O grau de instrução encontrado neste estudo foi considerado fator de risco, mulheres com menor grau de instrução, foram as mais acometidas com neonatos com defeitos congênitos, a orientação educacional é de extrema importância para a tomada de decisões cotidianas, os resultados encontrados neste estudo vão de encontro com outros artigos nacionais, como o de Fontoura FC e Cardoso MV (2014). Esta variável também indica o maior nível de escolaridade como fator protetor, uma vez que estas mães geralmente pertencem à uma seleta classe social, a qual tem acesso a serviços de saúde com maior facilidade e qualidade, também 
vale ressaltar, as questões alimentares, pois este grupo tende a ter hábitos alimentares saudáveis e também maior capacidade de interpretar possíveis riscos à saúde durante o período gestacional (ANDRADE AM, et al., 2017).

Nesta mesma linha de pensamento sobre determinantes sociais, se enquadra o estado cível da gestante, esta pesquisa mostrou que mulheres com situação conjugal estável obtém um padrão favorável ao bebê em relação as mulheres solteiras, separadas ou viúvas. Um estudo realizado na cidade de São Luís, em Maranhão, demonstrou este mesmo perfil que indicam que estas mulheres tendem a se sentir inseguras sem a presença do companheiro, e isto podem interferir nos fatores de riscos reprodutivos (RODRIGUES LS, et al., 2014).

Em relação as etnias, um estudo realizo na Região Centro Oeste brasileira, por Moraes CL, et al. (2020) encontrou resultados semelhantes a este estudo, onde mulheres pretas, indígenas e pardas, estão predispostas a gerar fetos com algum defeito congênito, podendo gerar uma hipótese ligada a fatores genéticos ou sociais, estando até o momento indefinido quais delas há maior influência sobre a ocorrência dos casos.

O parto e a gestação são variáveis geralmente apontadas como fatores de risco para a incidência de anomalia congênitas, entretanto, estes dados deves ser minuciosamente analisados e discutidos, visto que existem aspectos que limitam tais associações. A duração da gestação encontrada neste estudo apresentou risco para o período gestacional menor que 37 semanas, o que está em concordância com outros estudos realizados nos estados de São Paulo e no Acre (COSME HW et al., 2017; ANDRADE AM, et al., 2017). Isto, tem chances de estar relacionado as interrupções de gestações em casos do reconhecimento da anomalia congênita ainda no embrionário, da mesma forma, pode estar associado aos casos em que a respectiva anomalia seja um agravante para dar continuidade a gestação e acaba desencadeado o parto prematuro (JUNIOR EP, et al., 2017;).

A variável sobre o tipo parto não foi associada a ocorrência de anomalias congênitas nesta pesquisa, este dado está em desacordo com outros estudos, que justificam o parto cesáreo neste caso devido a AC e outros fatores predispostos da mãe (FONTOURA FC e CARDOSO MV, 2014; LUZ GS, et al., 2019).

A literatura descreve o período de pré-natal como um fator de grande importância na gestação, um estudo realizado por Luz GS, et al. (2019), amostrou que mães que aderem e realizam uma quantidade adequada de consultas de pré-natal são fator protetor quando analisada a associação com $A C$, o que vai de encontro com os resultados encontrados neste estudo (LUZ GS, et al., 2019).

Uma variável presente no estudo que não apresentou associação com a ocorrência de anomalias congênitas em Porto Alegre, foi o tipo de gestação que apresentou valor de $p$ de 0,384 , o que vai de encontro com outro estudo realizado em Tangará da Serra no Mato grosso (SILVA JH, et al., 2018)

Em relação ao peso do recém-nascido, geralmente é encontrado correlação com a incidência de anomalias congênitas, além de ser considerado a principal causa de mortalidade infantil pela OMS, o que ressalta que estes neonatos tendem a nascer mais debilitados devido à presença desta $A C$, o que pode vir a acarretar no atraso do desenvolvimento infantil. Diversas pesquisas indicam a prematuridade como uma característica dos nascidos vivos com alguma anomalia congênita (COSME HW, et al., 2017; SILVA JH, et al., 2018; ANDRADE AM, et al., 2017) este resultado também foi encontrado no presente estudo.

A escore de apgar é um método utilizado para realiza uma avaliação das condições de saúde no primeiro momento de vida, um estudo realizado no Rio Grande do Sul por Luz GS, et al. (2019) mostrou que os recémnascidos provenientes de alguma congênita, apresentam um score menor que 7 , o mesmo resultado encontrado dentro da cidade de Porto Alegre $(p<0,001)$ conforme o teste de qui-quadrado de Person realizado nesta pesquisa, podendo se constar que neonatos acometidos por alguma anomalia congênita, tendem a ter sua condição de saúde fragilizada (LUZ GS, et al., 2019).

A incidência de casos na cidade de Porto Alegre foi de 1,55 ao decorrer destes anos e vem apresentando declínio ao longo deste período, este resultado é positivo e promissor, após a análise estatística mostrar que 
a tendência é que o número de nascidos vivos diminua. No Brasil, foi implantado no ano de 2005, uma ação profilática de suplementação com ácido fólico para gestantes, por meio do Programa Nacional de Suplementação de Ferro (MINISTÉRIO DA SAÚDE, 2013).

Após a efetivação destas medidas, o percentual de anomalias congênitas demonstrou declínio, um estudo realizado no estado do Rio de Janeiro, na cidade de Campos dos Goytacazes, com o objetivo de obter as características epidemiológicas sobre as $\mathrm{AC}$ analisou os dados sobre a implementação do ácido fólico e atestou a redução das anomalias de defeitos do tubo neural em níveis satisfatórios, em um outro estudo de revisão integrativa, realizado por Silva CM, et al. (2020) reforçou a importância do uso da vitamina como auxiliar na prevenção dos casos de fenda palatina e fissuras orais (CRUZ CN, et al., 2019; SILVA CM, et al., 2019).

Os defeitos congênitos com maior prevalência neste estudo, estão de acordo com o último relatório emitido pela Agência Nacional de Vigilância Sanitária (ANVISA) onde foi realizado uma pesquisa semelhante e revelou que as anomalias congênitas com maior prevalência em território nacional foram malformações dos membros, sistema nervoso e órgãos genitais, outros estudos realizados em diferentes estados brasileiros, atestam estes mesmos dados (MINISTÉRIO DA SAÚDE, 2019; SIRTOLI DB, et al., 2017).

As malformações de membros, conforme Ministério da Saúde (2014) que em alguns casos o defeito pode ser reduzido com manobras manuais. Os defeitos congênitos do aparelho geniturinário são diagnosticados após as primeiras $24 \mathrm{~h}$ de vida, os exames de imagem são essenciais para que isso ocorra, por este motivo, é de extrema importância que este registro seja fidedigno e se for necessário recuperar estas DNVs para o correto preenchimento (SIRTOLI DB, et al., 2017).

Outro achado intrigante no estudo, é a diferença no número de casos de defeitos congênitos no sexo masculino quando comparamos ao feminino, que foi estatisticamente significativo. Nos resultados achados no município de Porto Alegre, há uma prevalência expressivamente maior no sexo masculino. O risco de anomalias de acordo com sexo do bebê é contraditório na literatura, embora alguns estudos apontem o sexo masculino, este fato parece estar restrito a somente alguns tipos de mal formações como fenda palatina e lábio leporino no sexo masculino e malformações congênitas dos membros no sexo feminino (SOKAL R, et al., 2014). Em vista disto, se faz necessário descobrir algum fator que esteja impedindo o desenvolvimento embriofetal e também estratificar melhor as anomalias registradas avaliando cada DNV individualmente com o objetivo de investigar com maior acuidade.

A realização do presente estudo conta com possíveis limitações devido aos dados terem sido extraídos de um banco de dados públicos, irrestrito, a confiabilidade e qualidade destes dados, dependem do preenchimento correto dos profissionais responsáveis pelos mesmos, o que ressalta a importância de capacitações e avaliações periódicas dos dados, a fim de buscar melhorias dentro do sistema e possíveis falhas, uma vez que o campo "ignorado" é muito utilizado, desvalorizando a informação e fazendo com que o sistema e pesquisas realizadas dentro dele, perca sua credibilidade muitas vezes.

\section{CONCLUSÃO}

Os fatores associados a prevalência de anomalias congênitas encontrados no presente foram: idade materna, sendo ela nos extremos (avançada e precoce), o baixo grau de instrução, mulheres solteiras e separadas das raças pretas, pardas e indígenas com gestações prematuras e uma adesão menor ao PN. Referente aos dados do recém-nascido, os dados encontrados foram pré-maturos com escore de apgar baixo. Considerando o perfil de anomalias congênitas, apresentaram-se com maior frequência, as AC do sistema osteomuscular, sistema circulatório e do aparelho genitourinário.

\section{REFERÊNCIAS}

1. AMERICAN HEART ASSOCIATION. Diagnosis and Treatment of Fetal Cardiac Disease. Circulation, 2014; 129(21): 2183-2242.

2. ANDRADE AM, et al. Anomalias congênitas em nascidos vivos. Revista Brasileira em Promoção a Saúde, 2017; 30(3): 1-11. 
3. BATEMAN BT, et al. Chronic hypertension in pregnancy and the risk of congenital malformations: a cohort study. Am J Obstet Gynecol, 2015; 212(3): 1-337.

4. COSME HW, et al. Garcia. Prevalência de anomalias congênitas e fatores associados em recém-nascidos do município de São Paulo no período de 2010 a 2014. Rev. paul. pediatr., São Paulo, 2017; 35(1): 33-38.

5. CRUZ CN, et al. Características epidemiógicas das anomalias congênitas do tubo neural em menores de 5 anos de idade e possíveis fatores de risco para sua ocorrência em Campos dos Goytacazes, Rio de Janeiro. Revista cientifíca da FMC. 2019; 14(1): 22-27.

6. FONSECA JJ. Metodologia da pesquisa científica. UEC, 2002. Apostila.

7. FONTOURA FC, CARDOSO MV. Associação das malformações congênitas com variáveis neonatais e maternas em unidades neonatais numa cidade do Nordeste brasileiro. Texto contexto - enferm, 2014; 23(4): 907-914.

8. FRANÇA E, LANSKY S. Mortalidade Infantil Neonatal no Brasil: Situações, Tendências e Perspectivas. Rede Interagencial de Informações para a Saúde. 2016; 24(4)4: 711-720.

9. JUNIOR EP, et al. Prevalência e fatores associados às anomalias congênitas em recém-nascidos. Revista Brasileira em Promoção a Saúde, 2017; 30(3): 1-9.

10. LUZ GS, et al. Anomalias congênitas no estado do Rio Grande do Sul: análise de série temporal. Rev. bras. Epidemiol., 2019; 22(1): 2-14.

11. LIMA L, et al. Perfil dos óbitos por anomalias congênitas no estado do Rio Grande do Norte no período de 2006 a 2013. Revista de Ciências Médicas e Biológicas de Salvador. 2017; 16(1): 52-58.

12. MENDES CM, et al. Anomalias congênitas e suas principais causas evitáveis: uma revisão. Revista médica de Minas Gerais. 2018; 28 e-1977; 2-6..

13. MINISTÉRIO DA SAÚDE. Departamento de Informática do Sistema Único de Saúde (DATASUS). Sistema de informações sobre nascidos vivos. Brasil, 2017. Disponível em: http://tabnet.datasus.gov.br/cgi/tabcgi.exe?sinasc/cnv/nvrs.def. Acesso em: 23 de setembro de 2020.

14. MINISTÉRIO DA SAÚDE. Saúde Brasil 2018 uma análise de situação de saúde e das doenças e agravos crônicos: desafios e perspectivas. Ministério da Saúde., Brasília, Brasil, 2019. Disponivel em: https://bvsms.saude.gov.br/bvs/publicacoes/saude_brasil_2018_analise_situacao_saude_doencas_agravos_cronico s_desafios_perspectivas.pdf. Acesso em: 12 de dezembro de 2020.

15. MINISTÉRIO DA SAÚDE. Programa Nacional de Suplementação de Ferro Manual de condutas gerais. Brasília, Distrito Federal, 2013. Disponivel em: https://bvsms.saude.gov.br/bvs/publicacoes/manual_suplementacao_ferro_condutas_gerais.pdf. Acesso em: 19 de novembro de 2020.

16. MINISTÉRIO DA SAÚDE. Situação das doenças e agravos crônicos, desafio e perspectivas. Ministério da Saúde. Brasília, DF, 2014.2 Disponivel em: https://bvsms.saude.gov.br/bvs/publicacoes/saude_brasil_2014_analise_situacao.pdf. Acesso em: 22 de novembro de 2020.

17. MORAES CL, et al. Frequency of Congenital Anomalies in the Brazilian Midwest and the Association with Maternal Risk Factors: Case-control Study. Rev. Bras. Ginecol. Obstet., 2020; 42(4): 188-193.

18. ORGANIZAÇÃO MUNDIAL DA SAÚDE (OMS). Registros de defeitos congênitos. Brasília. 2019 Disponível em: https://www.paho.org/bra/index.php?option=com_content\&view=article\&id=6005:registros-de-defeitos-congenitosestao-em-expansao-na-america-latina\&ltemid=820. Acesso em: 18 de dezembro de 2020.

19. RODRIGUES LS, et al. Características das crianças nascidas com malformações congênitas no município de São Luís, Maranhão, 2002-2011. Epidemiol. Serv. Saúde, 2014; 23(2) 295-304.

20. SILVA JH, et al. Perfil das anomalias congênitas em nascidos vivos de Tangará da Serra, Mato Grosso, $2006-2016$. Epidemiol. Serv. Saúde, 2018; 27(3): 1-10

21. SILVA CM, et al. O papel do ácido fólico na prevenção das fissuras labiopalatinas não sindrômicas: uma revisão integrativa. 2019; 3(1): 641-658.

22. SIRTOLI DB, et al. Sistema de Informação sobre Nascidos Vivos (SINASC): Uma fonte de dados sobre Anomalias congênitas nos recém-nascidos do município de Gurupi-Tocantins. Revista Cientifica do ITPAC, 2017; 10(2): 13-23.

23. SOKAL R, et al. Sex prevalence of major congenital anomalies in the United Kingdom: A national population-based study and international comparison meta-analysis. Clinical and Molecular Teratology. 2014; 100(2) 59-126. 